\title{
Oferta y demanda de los recursos turísticos Reserva Forestal Protectora Cerro Quininí (Tibacuy-Cundinamarca)
}

\author{
Offer and demand tourism resources Reserve Protective Forest Cerro Quinini \\ (Tibacuy-Cundinamarca)
}
Oferta e procura de recursos turísticos Reserva Florestal Protetora Cerro Quininí (Tibacuy-Cundinamarca)

\author{
Claudia Patricia Castellanos-Menjura ${ }^{1}$ William Ariza-Cortés ${ }^{2}$ William Fernando Castrillón-Cardona ${ }^{3}$
}

Recibido: Enero de 2019

Aceptado: Abril de 2019

Para citar este artículo: Castellanos-Menjura, C.P.; Ariza-Cortés, W.; Castrillón-Cardona, W.F. (2019). Oferta y demanda de los recursos turísticos Reserva Forestal Protectora Cerro Quininí (Tibacuy-Cundinamarca). Revista Científica, 36(3), 297-312. Doi: https://doi.org/10.14483/23448350.15075

\section{Resumen}

Para esta investigación se evaluaron los recursos turísticos y se caracterizó la demanda turística en las zonas de uso público de la Reserva Forestal Protectora del Cerro Quininí (RFPCQ), en la región de Sumapaz (Tibacuy, Cundinamarca, Colombia), con el fin de presentar un diagnóstico de la actividad turística en la zona y convertirse en el insumo para la planeación y concreción de un plan de manejo de la actividad turística. La metodología para la evaluación de la oferta frente a la gestión del turismo se realizó a través de entrevistas, caminatas y talleres con expertos locales y la comunidad de la zona, se obtuvo información para la identificación y clasificación de los recursos turísticos biofísicos, culturales y arqueológicos. Por otro lado, se evaluó la demanda de estos recursos con la aplicación de una encuesta para obtener el perfil de los visitantes, cuyos datos se analizaron por medio de la técnica de análisis de correspondencias múltiples que presentó información sobre tendencias y preferencias. La correlación entre la oferta de los recursos y la demanda se articularon en un análisis Dofa que permite la toma de decisiones frente al turismo como una estrategia de conservación y protección en el área de estudio. Con los resultados se obtiene el diagnóstico de la oferta y demanda turística, que sirve como base para el diseño del plan de manejo turístico orientado a las necesidades y conservación de la RFPCQ.

Palabras clave: recursos turísticos, análisis multicriterio, área protegida, toma de decisiones, visitante.

\footnotetext{
Abstract

Tourist resources were evaluated and tourist demand was characterized in the areas of public use of the Cerro Quininí Protective Forest Reserve (RFPCQ), in the Sumapaz region (Tibacuy,
} 
Cundinamarca, Colombia), in order to present a diagnosis of tourism activity in the area and become the input for the planning and implementation of a management plan for tourism. The methodology for the evaluation of the offer in relation to tourism management was carried out through interviews, walks and workshops with local experts and the community of the area. Information was obtained for the identification and classification of biophysical, cultural and tourism resources. archaeological On the other hand, the demand for these resources was evaluated with the application of a survey to obtain the profile of the visitors whose data were analyzed by means of the Multiple Correspondence Analysis techniques that presented information on trends and preferences. The correlation between the supply of resources and demand was articulated in a SWOT analysis that allows decision-making regarding tourism as a conservation and protection strategy in the study area. With the results, the diagnosis of the tourist supply and demand is obtained, which serves as the basis for the design of the tourism management plan oriented to the needs and conservation of the RFPCQ.

Keywords: tourism resources, multicriteria analysis, protected area, decision making, visitor.

\section{Resumo}

Recursos turísticos foram avaliados e caracterizados demanda turística em áreas de uso público da protecção Reserva Florestal de Cerro Quininí (RFPCQ), na região de Sumapaz (Tibacuy, Cundinamarca, Colômbia), a fim de apresentar um diagnóstico de atividade turística na área e se tornar o insumo para o planejamento e implementação de um plano de manejo para o turismo. A metodologia de avaliação de abastecimento contra a gestão do turismo foi conduzida por meio de entrevistas, passeios e workshops com especialistas locais e comunidade da região, informações para a identificação e classificação dos recursos turísticos biofísicas, culturais são obtidas e arqueológico Por outro lado, a demanda por esses recursos foi avaliada com a aplicação de uma pesquisa para obter o perfil dos visitantes cujos dados foram analisados por meio da técnica de Análise de Correspondências Múltiplas, que apresentou informações sobre tendências e preferências. A correlação entre oferta de recursos e demanda foi articulada em uma análise SWOT que permite a tomada de decisão em relação ao turismo como estratégia de conservação e proteção na área de estudo. Com os resultados, obtém-se o diagnóstico da oferta e demanda turística, que serve de base para o desenho do plano de manejo turístico orientado às necessidades e conservação do RFPCQ.

Palavras-chaves: recursos turísticos, análise múltipla, área protegida, toma de decisões, visitante

\section{Introducción}

El turismo es uno de los mercados más dinámicos del mundo del cual se obtienen múltiples beneficios económicos, socioculturales y ambientales; se trata, pues, de una actividad económica basada en la oferta y la demanda de recursos turísticos (Boullón, 1978). La Organización Mundial del Turismo (OMT, 1998) establece como líneas especializadas del turismo: el turismo de aventura, el agroturismo, el turismo de cruceros, el turismo cultural y el ecoturismo. En Colombia, el ecoturismo se ha concentrado especialmente en áreas protegidas del Sistema de Parques Nacionales y la Red de Reservas de Sociedad Civil, que toma relevancia e importancia a partir de la Ley 300 de 1996, reglamentada por el Decreto Nacional 2590 de 2009, como una de las líneas especializadas de turismo (Ministerio de Comercio Industria y Turismo-MCIT, 2013). Es una estrategia de conservación para la mejora y mantenimiento de los valores naturales y culturales en las áreas protegidas, tiene como fin la articulación de la diversidad biológica con lo social a partir del conocimiento y disfrute del patrimonio natural de los visitantes. Requiere de una regulación para la planificación que prevea el proceso de análisis del sistema, planeación, ordenamiento, implementación y seguimiento en las áreas protegidas (Cubillos et al., 2013).

Para iniciar un proceso de planeación turística es necesaria la localización y verificación objetiva 
del potencial de la actividad de un destino turístico en una determinada localidad, municipio o región considerado como un "territorio" o "espacio turístico" (Sectur, 2005), en el que se ofrezcan diversos productos, se consolide el mercado turístico y se concreten espacios con atractivos potenciales que integren la demanda y la oferta (Angulo, 2002; Jiménez-Bulla y Jiménez-Barbosa, 2013).

Las fases previstas en la planeación de la actividad turística son: diagnóstico, ordenamiento y plan de acción. En el diagnóstico se establece la situación actual de un área protegida, su zona de influencia y las tendencias a partir de información que se obtiene de manera directa o secundaria; con ello se busca conocer la dinámica local e integrarla en la planificación regional (Dredge, 1999), identificar y localizar los atractivos, las actividades y servicios asociados al ecoturismo, el perfil del visitante, el nivel de satisfacción y realizar un análisis situacional que permitan identificar líneas de acción prioritarias. En el ordenamiento se diseña la experiencia del visitante y las herramientas que le permitan el óptimo manejo, regulación y control del ecoturismo. El plan de acción corresponde a las diferentes líneas de acción que orientarán el ecoturismo en una determinada área protegida con las respectivas metas, actividades, responsables, tiempos de ejecución y recursos (Cubillos et al., 2013).

En los últimos años la Reserva Forestal Protectora Cerro Quininí (RFPCQ), considerada como una zona que debe ser conservada permanentemente con bosques naturales o artificiales para proteger estos mismos recursos $u$ otros naturales renovables (art. 204, Decreto 2811, 1974), se ha transformado es un espacio turístico muy visitado por personas que se desplazan de poblaciones cercanas y del distrito capital de Bogotá. La importancia que ha tomado radica en la riqueza paisajística, cultural, arqueológica y natural. Los ecosistemas que se encuentran allí se caracterizan por facilitar la conectividad con otras áreas protegidas como Peñas Blancas, Salto del Tequendama y Cerro Majuy (AIcaldía de Tibacuy, s. f.).
El problema, tema de estudio de esta investigación, se enfoca en que las actividades turísticas que se practican en la RFPCQ se adelantan sin un plan de manejo (Corporación Autónoma Regional-CAR, 2012). Por esta razón, es pertinente que los visitantes cuenten con una adecuada orientación de las actividades que se puedan realizar. Esto, sumado con la transformación del uso del suelo por parte de las comunidades locales, lo que ha causado la disminución considerable de los bosques y, por ende, el deterioro de los ecosistemas que allí se encuentran (Ministerio del Medio Ambiente y Desarrollo Sostenible, 2012). El presente documento se enmarca en la fase diagnóstica de la planificación turística, en la que primero se realiza la identificación, evaluación y potencialidad de la oferta de los recursos turísticos a partir de la caracterización de los atributos con énfasis en los criterios intrínsecos y extrínsecos; segundo, con la demanda y el análisis del perfil del visitante; y, tercero, con la correlación entre la oferta de los recursos turísticos y la demanda articulados en un análisis Dofa que permita la toma de decisiones frente al ecoturismo como una estrategia de conservación en el área de estudio.

\section{Metodología}

La investigación se llevó a cabo en los andes colombianos, en la cordillera oriental, en el área protegida denominada RFPCQ, en el municipio de Tibacuy, Cundinamarca, Colombia, ubicada a los $04 .^{\circ} 19^{\prime}$ de latitud norte y $74 .^{\circ} 30^{\prime}$ de longitud oeste. Se encuentra en un intervalo altitudinal de entre 1050 y 2100 msnm, la temperatura promedio de $21^{\circ} \mathrm{C}$, la superficie es de aproximadamente 1947 ha, en las que se hallan comprometidos los municipios de Tibacuy $(1571,71$ ha), Viotá (21,50 ha) y Nilo (173,90 ha). Esta área fue declarada por el Inderena, mediante el acuerdo 029 de 1987, y aprobada, mediante resolución n. ${ }^{\circ} 122$ de 1987, por el Ministerio de Agricultura como Área de Reserva Forestal Protectora. 
La geología se caracteriza por tener formaciones sedimentarias del cretáceo y del terciario. Las características como la litología y la disposición de las capas son evidencia de la evolución geológica de la cordillera oriental, con grandes capas de sedimentos marinos y continentales elevados por la orogenia andina (Salas y Tapias, 2000). Ubicada en la zona de vida de bosque húmedo premontano (bh-PM), una menor proporción pertenece al bosque seco premontano (bs-PM) (Holdridge, 1947). Más del $90 \%$ de los predios de la RFPCQ son de carácter privado y están utilizados en cultivos y pastos, siendo el cultivo de café el que mayor espacio ocupa. La actividad económica se centra en la explotación forestal, el cultivo de frutas y el turismo como una alternativa emergente (Vásquez, 2005).

\section{Identificación y evaluación del potencial de la oferta de los recursos turísticos}

Para la identificación y ubicación de los recursos turísticos se digitalizó un mapa en ArcView GIS 3.2 a una escala de 1:10 000, en el que se trazaron las zonas de uso público que los visitantes normalmente siguen. Las planchas base se ubicaron en el Instituto Geográfico Agustín Codazzi (Igac). Se identificaron las coberturas de la RFPCQ, lo que permitió presentar una descripción desde lo físico y ver la potencialidad turística.

En cuanto la metodología para la toma y procesamiento de datos, se usó la técnica de Evaluación Multicriterio para evaluar la oferta de los recursos turísticos, se identificó su potencialidad a partir de caracterización de los atributos con énfasis en los criterios intrínsecos y extrínsecos de acuerdo con las recomendaciones de (Sectur, 2004; Zamorano, 2002). La información de la oferta turística se obtuvo a partir de sesiones de trabajo con los expertos locales quienes identificaron los lugares y las áreas de ubicación estos recursos, realizando recorridos de campo y talleres. En esta investigación se integran los datos espaciales con las percepciones de los expertos locales (Jarvis et al., 2006; Seixas,
2005). El Procedimiento para el análisis de Evaluación Multicriterio (EMC) ${ }^{4}$ incluyó: a) definición del objetivo e identificación de alternativas; b) definición del conjunto de atributos: criterios intrínsecos y criterios extrínsecos; c) obtención de la matriz de decisión y; d) valoración de los recursos turísticos mediante la aplicación del método de sumatoria lineal ponderada.

\section{Perfil del visitante}

El perfil del visitante forma parte de la fase diagnóstica para la planificación del ecoturismo $(\mathrm{Cu}-$ billos, 2013) de la RFPCQ y consta de cuatro momentos: 1) obtención de la muestra de la demanda turística; 2) desarrollo de la encuesta en un cuestionario de 31 preguntas aplicado a 111 personas para determinar el perfil del visitante $y$ definir sus preferencias de acuerdo con los segmentos que caracterizaron el instrumento; 3) análisis de los datos, los cuales se procesaron en el programa SPSS $(19,0)$ luego transferidos al paquete estadístico SPAD. Este último, permite realizar análisis de perfiles a partir del análisis de correspondencias múltiples, cuyo objetivo es resumir muchos datos en un número reducido de dimensiones con la menor pérdida de información (De la Fuente, 2011).

\section{Análisis DOFA}

Forma parte de la fase de planificación para definir las estrategias. A partir de ella se identificaron las fortalezas, oportunidades, debilidades y amenazas que se articulan a los resultados obtenidos en la identificación de la oferta de los recursos turísticos y la demanda según el perfil del visitante. Esta técnica es muy utilizada dentro del proceso de planificación estratégica, pues es una de las herramientas que permite hacer el análisis de las

\footnotetext{
Franco-Maass et al. (2009). Evaluación multicriterio de los recursos turísticos: Parque Nacional Nevado de Toluca. Estudios y Perspectivas en Turismo, 18, 208-226 P.7
} 
variables que, entre otras, componen un territorio (Torrejón, 2006).

\section{Resultados}

\section{Coberturas y ubicación de la oferta de los recur- sos turísticos}

Se identificaron cuatro coberturas: bosques, tierras eriales, cultivos y vegetación herbácea descubierta, las cuales se evidencian en el mapa de cobertura del sendero ecoturístico ubicado en la RFPCQ, Cundinamarca, Colombia (figura 1). Los bosques ocupan un área representativa con un estrato superior de hasta $30 \mathrm{~m}$, presenta especies de flora principalmente heliófitas, características de bosques secundarios. De los registros realizados en la zona, según Pinzón (2010), se destacan especies como cedrillo (Brunellia comocladifolia), caucho (Ficus trigona), puntelanza (Vismia baccifera), amarillos (Ocotea spp. Nectandra spp.), tinto (Palicorea spp.), Tunos (Miconia minutiflora), garrocho (Viburnum cornifolium), gaque (Clusia multiflora), amarillo jigua (Nectandra membranacea), quasio (Picrasma excelsa), pedro hernández (Toxicodendron striatum).

Se han registrado 27 especies de orquídeas, muchas de ellas con alto valor estético e importantes en bioprospección (Ordoñez y Montes, 2013). En algunas áreas de la parte alta se destaca la presencia de individuos de roble (Quercus humboldtii) conformando asociaciones denominadas robledales, categorizada como una especie Vulnerable (VU), según el libro rojo de plantas de Colombia (Cárdenas y Salinas, 2007). También se encuentran especies que son poco frecuentes pero importantes para la conservación debido a su rareza y por ser remanentes de los bosques primarios, tales como Meliosma frondosa, Andira taurotesticulata y Pouteria torta (Pinzón, 2010); por lo que se deben generar estrategias para su manejo y conservación. Se registra también el arbusto Notopleura cundinamarcana, endémico de la provincia del Sumapaz. Si bien no existen estudios detallados sobre la fauna, en la información de los pobladores locales se advierte la presencia de numerosas especies de aves y mamíferos pequeños sobre los cuales se podrían desarrollar actividades de interpretación que generen un mayor atractivo turístico para la reserva; se destacan las especies de aves por su diversidad, colorido y frecuencia a lo largo de los senderos.

Los cultivos que interactúan con los ecosistemas de bosques dan lugar a los agroecosistemas. Se evidencia, a lo largo de los recorridos de la RFPCQ, la modificación de los ecosistemas por la actividad antrópica relacionada con los diferentes procesos de producción agrícola (Hernández, 1977).

Las tierras eriales son una cobertura en la que la superficie de la tierra no tiene capacidad para ser cultivada y labrada; se identifican como rocas expuestas y suelos desnudos (Vargas, 1992). Encierran un valor económico, escénico, científico o técnico. Presenta especial importancia por su imponente posición en la formación montañosa como afloramientos rocosos que sirven de escenario para la contemplación paisajística. En estas zonas se desarrollan deportes extremos como la escalada o el rapel. Tienen vegetación incipiente sobre la superficie que no oculta la morfología rocosa, entre ellas se encuentra la Mammillaria columbiana y la Sobralia purpurea, especies endémicas de esta cobertura (Pinzón, 2010). Es de destacar que algunos de los atractivos turísticos más importantes se encuentran en estas formaciones.

La vegetación herbácea descubierta es una cobertura en crecimiento en la que algunas especies de árboles comparten espacios con hierbas y pastos, en ocasiones están solos o en pequeños grupos y su finalidad es brindar forraje al ganado, principalmente vacuno doble propósito.

\section{Recursos turísticos con vocación ecoturística}

Se identificaron 19 recursos turísticos que se clasificaron en biofísicos, culturales y arqueológicos. A cada uno se le realizó una ficha de inventario del 
recurso turístico con la siguiente información: generalidades, características y total puntaje de valoración. Se les atribuyó criterios intrínsecos (tabla 1) y criterios extrínsecos (tabla 2).

En la tabla 3 se destacan las características de los criterios intrínsecos de los recursos biofísicos, la flora es moderada en la mayoría a excepción de los recursos turísticos denominados Bosque de Robles, los paisajes de las vistas panorámicas la meseta de Chinauta y del valle del Magdalena, en las que se observa que es abundante. Con respecto a la fauna, los expertos locales y la misma comunidad indican que no se han realizado estudios de fondo, excepto las aves y algunos mamíferos; sin embargo, en lo que conocen consideran que la fauna asociada con la mayoría de los recursos turísticos es moderada y escasa en aquellos que se encuentran en áreas de vegetación herbácea caracterizadas por agroecosistemas. En cuanto el relieve los recursos turísticos la Peña-Cerro Quininí, el Pico del Águila, la Cabeza del Indio, se encuentran en la cobertura de tierras eriales en áreas muy inclinadas. La Cueva del Mohán ubicada en el recurso turístico Ilamado Paisaje: Vista Meseta de Chinauta se caracteriza por estar en áreas muy inclinadas que son aprovechadas por los visitantes en prácticas de deportes extremos. Aquellos que se encuentran en áreas menos inclinadas son atractivas para realizar caminatas. La cobertura bosques es la más representativa, se encuentra asociada a aquellos recursos en los que se practica el senderismo o caminatas, se destacan el Bosque de Robles, Paisaje: Vista Meseta de Chinauta, Senderismo Antenas, Paisaje: Vista Panorámica Valle del Magdalena, Cueva del Mohán y Casa de Turismo: "La Cumbre".

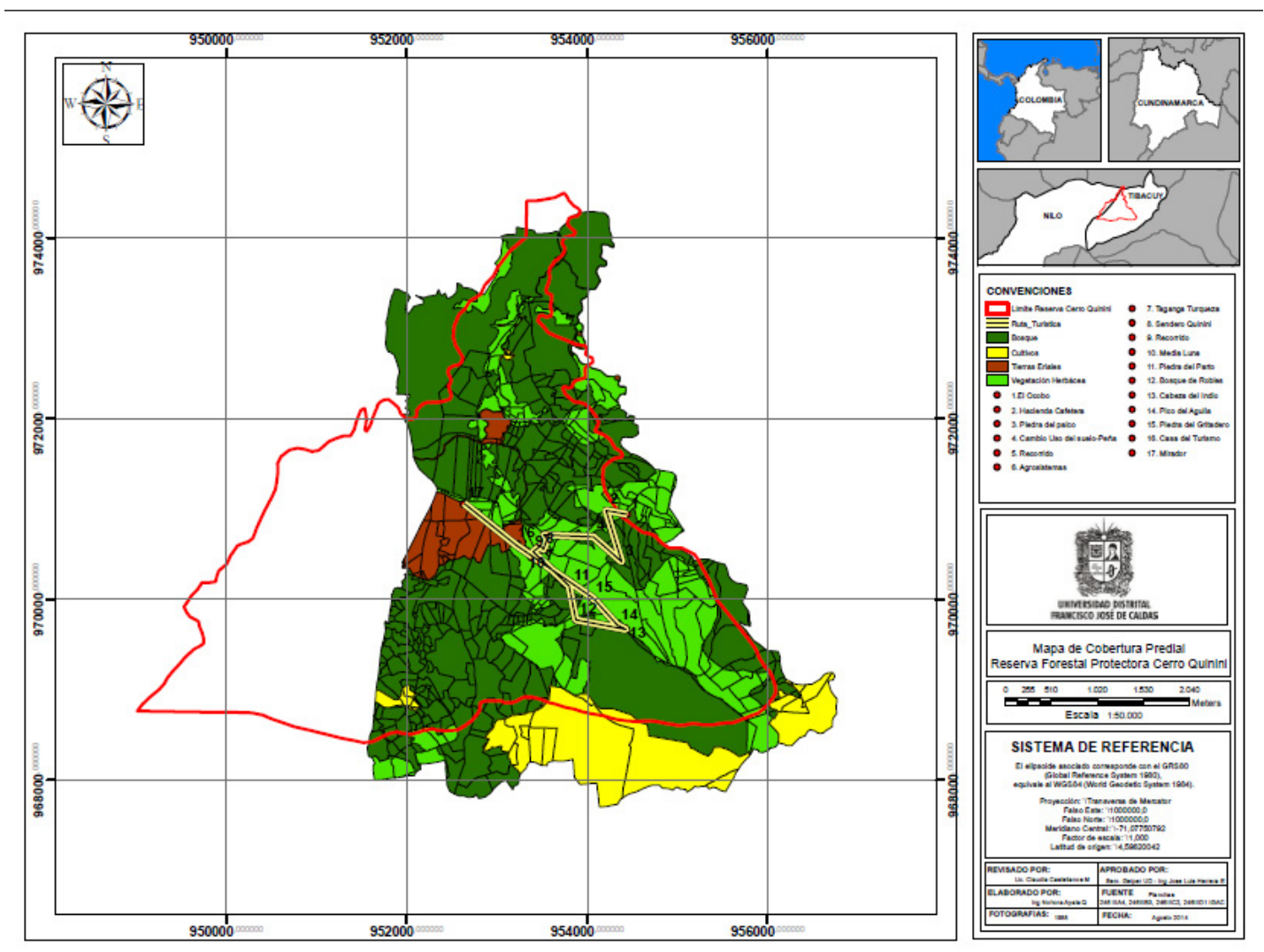

Figura 1. Mapa de coberturas del sendero turístico ubicado en la RFPCQ Cerro Quininí, Cundinamarca, Colombia. Fuente: elaboración propia a partir mapa predial y fotografías aéreas IGAC. 
Tabla 1. Criterios intrínsecos de los recursos biofísicos, culturales y arqueológicos.

\begin{tabular}{|c|c|c|c|c|}
\hline Tipo de Recurso & Criterio & Atributo & Descripción & Símbolo \\
\hline \multirow{23}{*}{ Biofísicos } & \multirow{6}{*}{ a) Diversidad } & \multirow{3}{*}{ a1) Flora } & Abundante & (A) \\
\hline & & & Suficiente & $(\mathrm{S})$ \\
\hline & & & Escasa & $(\mathrm{E})$ \\
\hline & & \multirow{3}{*}{ a2) Fauna } & Abundante & (A) \\
\hline & & & Suficiente & $(\mathrm{S})$ \\
\hline & & & Escasa & (E) \\
\hline & \multirow{11}{*}{$\begin{array}{l}\text { b) Condiciones } \\
\text { del terreno }\end{array}$} & \multirow{4}{*}{ b1) Relieve } & Muy Inclinado & $(\mathrm{MI})$ \\
\hline & & & Inclinado & (I) \\
\hline & & & Algo Inclinado & $(\mathrm{Al})$ \\
\hline & & & Plano & $(\mathrm{P})$ \\
\hline & & \multirow{4}{*}{ b2) Cobertura } & Bosques & (Bo) \\
\hline & & & Cultivos & (C) \\
\hline & & & Tierras eriales & $(\mathrm{Te})$ \\
\hline & & & Vegetación herbácea & $(\mathrm{Vh})$ \\
\hline & & \multirow{3}{*}{ b3) Dificultad } & Alto & (A) \\
\hline & & & Medio & (M) \\
\hline & & & Bajo & (B) \\
\hline & \multirow{6}{*}{ c) Paisajes } & \multirow{3}{*}{$\begin{array}{l}\text { c1) Escala (Tamaño de Visión } \\
\text { del Observador) }\end{array}$} & Grande & (G) \\
\hline & & & Mediano & (M) \\
\hline & & & Pequeño & $(\mathrm{P})$ \\
\hline & & \multirow{3}{*}{$\begin{array}{c}\text { c2) Relación Hombre } \\
\text { Naturaleza }\end{array}$} & Impacto Positivo & (IP) \\
\hline & & & Impacto Neutro & $(\mathrm{IN})$ \\
\hline & & & Impacto Negativo & $(\mathrm{INe})$ \\
\hline \multirow{8}{*}{$\begin{array}{l}\text { Culturales y } \\
\text { arqueológicos }\end{array}$} & \multirow{4}{*}{$\begin{array}{l}\text { d) Interés } \\
\text { arqueológico }\end{array}$} & \multirow{4}{*}{ d1) Petroglifo } & Excelente & $(\mathrm{E})$ \\
\hline & & & Bueno & (B) \\
\hline & & & Regular & $(\mathrm{R})$ \\
\hline & & & Malo & $(\mathrm{M})$ \\
\hline & \multirow{4}{*}{ e) Fincas Cafeteras } & \multirow{4}{*}{ e1) Practica de producción } & Muy Activo & $(\mathrm{MA})$ \\
\hline & & & Activo & (A) \\
\hline & & & Poco Activo & $(\mathrm{PA})$ \\
\hline & & & Abandonado & (A) \\
\hline
\end{tabular}

Fuente: elaboración propia a partir de Enríquez, Osorio, Franco-Maass, Ramírez y Nava (2010) y Franco-Maass et al. (2009).

Tabla 2. Criterios extrínsecos de los recursos biofísicos, culturales y arqueológicos.

\begin{tabular}{|c|c|c|c|}
\hline Criterio & Atributo & Descripción & símbolo \\
\hline \multirow{2}{*}{ f) Acceso físico } & f1) Distancia & Kilómetros & \\
\hline & f2) Tiempo & Minutos & \\
\hline \multirow{11}{*}{ g) Apreciación } & \multirow{4}{*}{ g1) Estado de conservación } & Excelente & $(\mathrm{E})$ \\
\hline & & Bueno & (B) \\
\hline & & Regular & $(\mathrm{R})$ \\
\hline & & Malo & $(\mathrm{M})$ \\
\hline & \multirow{3}{*}{ g2) Calidad del entorno } & Optima & $(\mathrm{O})$ \\
\hline & & Buena & (B) \\
\hline & & Regular & $(\mathrm{R})$ \\
\hline & \multirow{4}{*}{ g3) Interés } & Grande & $(\mathrm{G})$ \\
\hline & & Alguno & (A) \\
\hline & & Poco & $(\mathrm{P})$ \\
\hline & & Ninguno & $(\mathrm{N})$ \\
\hline
\end{tabular}


Oferta y demanda de los recursos turísticos Reserva Forestal Protectora Cerro Quininí (Tibacuy-Cundinamarca) Castellanos-Menjura, C.P.; Ariza-Cortés, W.; Castrillón-Cardona, W.F.

\begin{tabular}{|c|c|c|c|}
\hline Criterio & Atributo & Descripción & símbolo \\
\hline \multirow{6}{*}{ g) Apreciación } & \multirow{4}{*}{ g4) Fragilidad } & Muy Alta & (MA) \\
\hline & & Alta & $(\mathrm{A})$ \\
\hline & & Moderada & $(\mathrm{M})$ \\
\hline & & Baja & ( Ba) \\
\hline & \multirow{2}{*}{ g5) Contaminación } & $\mathrm{Si}$ & $(\mathrm{S})$ \\
\hline & & No & $(\mathrm{N})$ \\
\hline \multirow{12}{*}{ h) Infraestructura y servicios } & \multirow{4}{*}{ h1) Señalización } & Suficiente & $(\mathrm{S})$ \\
\hline & & Alguna & $(\mathrm{A})$ \\
\hline & & Insuficiente & (I) \\
\hline & & Ninguno & $(\mathrm{N})$ \\
\hline & \multirow{4}{*}{ h2) Equipamiento } & Suficiente & $(\mathrm{S})$ \\
\hline & & Alguno & $(\mathrm{A})$ \\
\hline & & Insuficiente & (I) \\
\hline & & Ninguno & $(\mathrm{N})$ \\
\hline & \multirow{4}{*}{$\begin{array}{l}\text { h3) Actividades/rapel/caminatas } \\
\text { ecológicas/camping }\end{array}$} & Muy Organizadas & $(\mathrm{MO})$ \\
\hline & & Organizadas & $(\mathrm{O})$ \\
\hline & & Poco Organizada & (PO) \\
\hline & & Sin Organización & $(\mathrm{SO})$ \\
\hline \multirow{3}{*}{ i) Seguridad } & \multirow{3}{*}{ i1) Vigilancia } & Muy seguro & $(\mathrm{M})$ \\
\hline & & Seguro & $(\mathrm{S})$ \\
\hline & & Moderado & (Mo \\
\hline
\end{tabular}

Fuente: elaboración propia a partir de Enríquez et al. (2010) y Franco-Maass et al. (2009).

Tabla 3. Matriz recursos biofísicos, culturales y arqueológicos: características cualitativas de criterios intrínsecos.

\begin{tabular}{|c|c|c|c|c|c|c|c|c|c|}
\hline \multirow{3}{*}{ RECURSOS } & \multicolumn{9}{|c|}{ CRITERIOS INTRINSECOS } \\
\hline & $\begin{array}{l}\underset{v}{0} \\
\underset{\sqcup}{0}\end{array}$ & 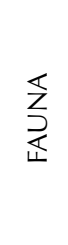 & 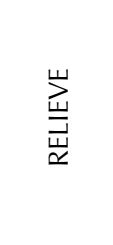 & 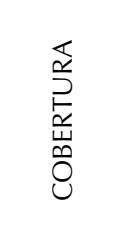 & 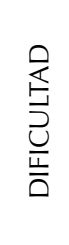 & 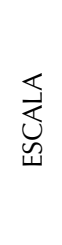 & 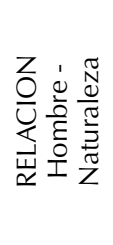 & 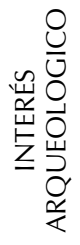 & 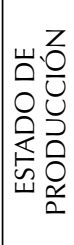 \\
\hline & $\begin{array}{l}\text { a1 } \\
(1)^{*}\end{array}$ & $\begin{array}{l}\text { a2 } \\
(2)^{*}\end{array}$ & $\begin{array}{l}\text { b1 } \\
(3)^{*}\end{array}$ & $\begin{array}{l}\text { b2 } \\
(4)^{*}\end{array}$ & $\begin{array}{l}\text { b3 } \\
(5)^{*}\end{array}$ & $\begin{array}{l}\mathrm{c} 1 \\
(6)^{*}\end{array}$ & $\begin{array}{l}\mathrm{C} 2 \\
(7)^{*}\end{array}$ & $\begin{array}{l}\mathrm{d} 1 \\
(8)^{*}\end{array}$ & $\begin{array}{l}\mathrm{e} 1 \\
(9)^{*}\end{array}$ \\
\hline \multicolumn{10}{|l|}{ BIOFISICOS } \\
\hline Peña - Cerro Quininí & Mo & Mo & $\mathrm{MI}$ & $\mathrm{Te}$ & Mo & G & IN & ---- & ---- \\
\hline $\begin{array}{l}\text { Recorrido: Cambio } \\
\text { uso del suelo }\end{array}$ & E & E & MIXTO & MIXTO & Mo & M & IN & --- & --- \\
\hline $\begin{array}{l}\text { Paisaje: Vista Meseta } \\
\text { de Chinauta } \\
\end{array}$ & A & $\mathrm{E}$ & $\mathrm{Al}$ & Bo & B & G & IN & ---- & ---- \\
\hline Agroecosistemas & Mo & Mo & $\mathrm{Al}$ & MIXTO & Mo & $M$ & IN & ---- & ---- \\
\hline $\begin{array}{l}\text { Senderismo: } \\
\text { Recorrido Fauna } \\
\text { y Flora - Tangara } \\
\text { Turquesa } \\
\end{array}$ & Mo & Mo & 1 & Bo & Mo & M & IN & ---- & ---- \\
\hline \begin{tabular}{|l|} 
Sendero: Cerro \\
Quininí \\
\end{tabular} & Mo & Mo & I & Bo & Mo & $P$ & IP & ---- & ---- \\
\hline Senderismo Antenas & Mo & Mo & $\mathrm{Al}$ & Bo & Mo & $M$ & $\mathrm{INe}$ & ---- & $\begin{array}{ll}--- \\
--\end{array}$ \\
\hline Bosque de Robles & $\mathrm{A}$ & $\mathrm{A}$ & $\mathrm{Al}$ & Bo & Mo & $M$ & $\mathrm{INe}$ & ---- & ---- \\
\hline Cabeza del Indio & Mo & Mo & $\mathrm{MI}$ & $\mathrm{Te}$ & Mo & $G$ & $\mathrm{INe}$ & $\begin{array}{ll}--- \\
\end{array}$ & $\begin{array}{ll}---- \\
\end{array}$ \\
\hline Pico del Águila & Mo & $E$ & $\mathrm{MI}$ & $\mathrm{Te}$ & $\mathrm{A}$ & $G$ & IN & ---- & ---- \\
\hline
\end{tabular}

Continúa 


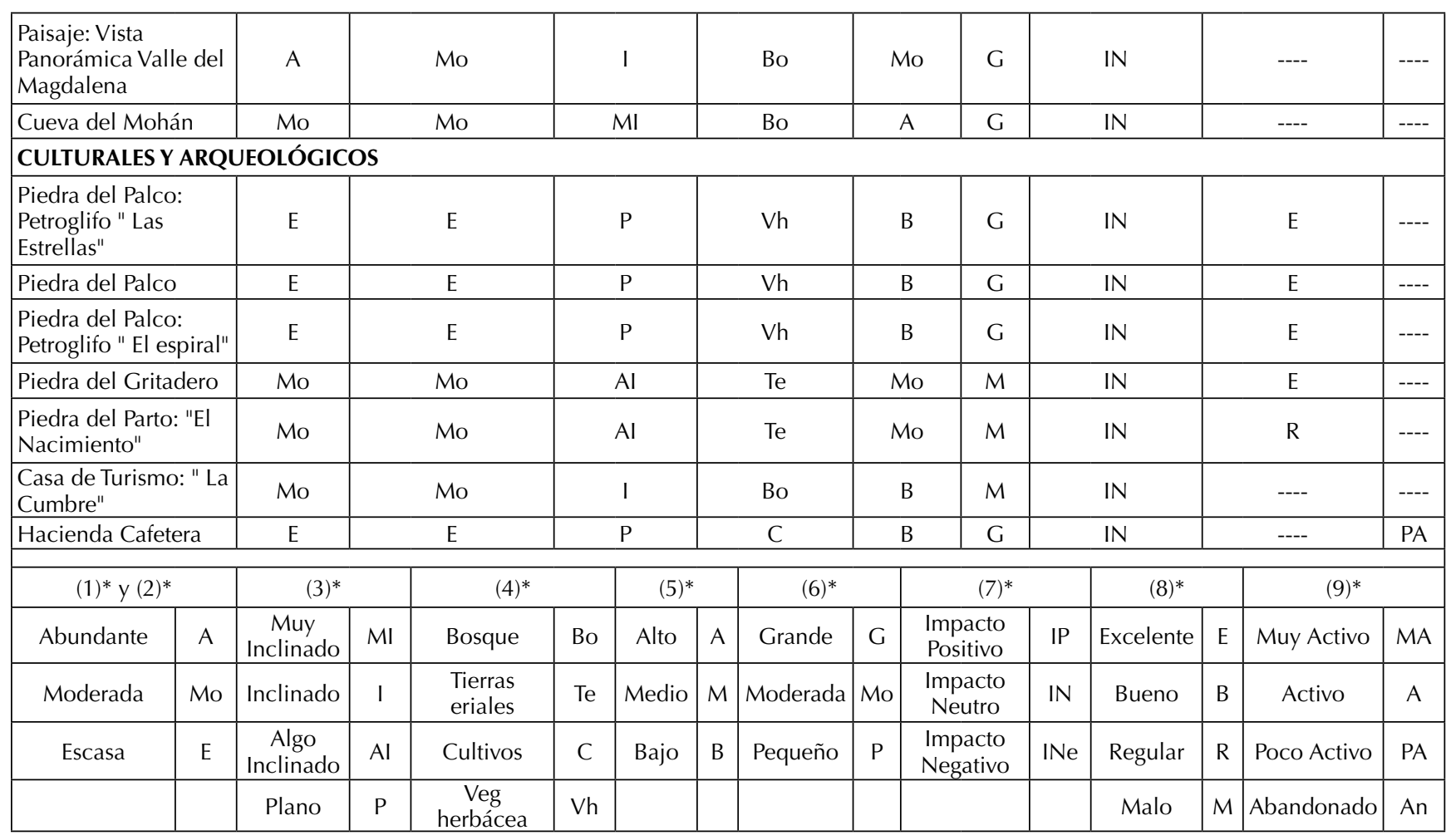

Fuente: elaboración propia a partir de Enríquez et al. (2010) y Franco-Maass et al. (2009).

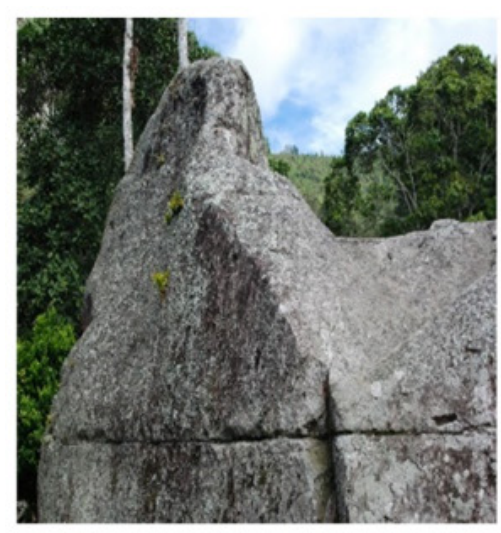

a. Piedra del Palco

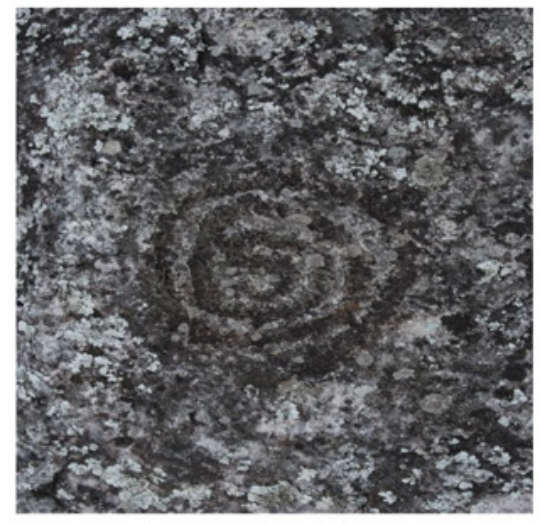

b. Petroglifo "El espiral"

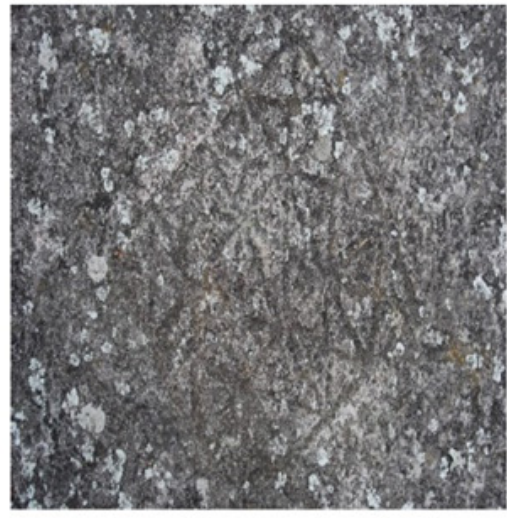

c. Petroglifo "Las Estrellas"

Figura 2. Recursos turísticos ubicados en el Piedra del Palco petroglifos "El espiral y las Estrellas".

Fuente: elaboración propia recorrido de reconocimiento Piedra del Palco, fotografías Mauricio Castro.

Por otra parte, los criterios intrínsecos para los recursos arqueológicos se caracterizan por encontrasen en relieve plano, la fauna y la flora que los rodea es escasa; se encentran en áreas de cobertura de vegetación herbácea, tierras eriales, bosque y cultivos. La dificultad para acceder se encuentra entre baja y moderada. Presentan un excelente grado de interés por parte de los turistas, quienes desean conocer lo relacionado con la cultura ancestral Panche. En la figura 2 se observa la piedra del palco (a), petroglifo "El Espiral" (b) y petroglifo "Las Estrellas" (c). 
En cuanto a la relación hombre-naturaleza es importante resaltar que hay un impacto negativo en el área de estudio debido a la actividad turística que va en constante crecimiento y el cual no presenta un adecuado manejo. De igual forma, el cambio del uso del suelo por parte de los lugareños afecta recursos turísticos dentro de los cuales se destacan los denominados Senderismo Antenas, Bosque de Robles y Cabeza del Indio. También se registró un impacto positivo en el recurso turístico denominado Sendero: Cerro Quininí, en el cual se ha trabajado en señalización y adecuación de la vía de acceso.

En la tabla 4 se muestran los criterios extrínsecos de los recursos turísticos biofísicos, culturales y arqueológicos. En ella se evidencia que el estado de conservación oscila entre bueno y regular; la calidad del entorno en general se consideró buena, el interés de parte de los turistas por visitar recursos resultó ser muy grande, considerando que las distancias son cortas para aquellos visitantes que se desplazan desde Bogotá. Desde Silvania hay trayectos a cada uno de los recursos turísticos que van desde 16 a $22 \mathrm{Km}$ y el tiempo de Ilegada oscila entre 60 y 160 minutos, la distancia y el tiempo se asocian en el desplazamiento que se hace en carro y caminando. En cuanto al manejo de los recursos turísticos que se ofertan, de manera empírica, los expertos locales manifestaron que, al no tener una estrategia de conservación en un plan de manejo, los recursos se encuentran en constate deterioro lo que hace que su fragilidad sea muy alta. En cuanto a la infraestructura para la atención de los turistas, en general se observó que es muy poca y los servicios que se prestan para la atención son insuficientes al no existir un equipamiento, actividades organizadas para el conocimiento de los recursos turísticos y del área protegida en general. Por último, en términos de seguridad se registró que esta es moderada, pues se realiza por parte de la comunidad y no hay vigilancia privada.

Tabla 4. Matriz recursos biofísicos, culturales y arqueológicos características cualitativas de criterios extrínsecos.

\begin{tabular}{|c|c|c|c|c|c|c|c|c|c|c|c|}
\hline \multirow{3}{*}{ RECURSOS } & \multicolumn{11}{|c|}{ CRITERIOS EXTRINSECOS } \\
\hline & \multirow{2}{*}{ 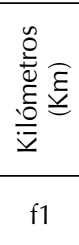 } & \multirow{2}{*}{ 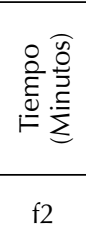 } & \multicolumn{5}{|c|}{$\begin{array}{l}\frac{0}{U} \\
\frac{\pi}{U} \\
\frac{0}{2} \\
\frac{0}{2}\end{array}$} & \multicolumn{2}{|c|}{ 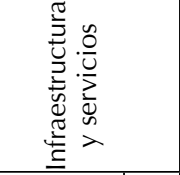 } & \multicolumn{2}{|c|}{ 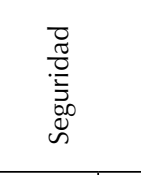 } \\
\hline & & & $\begin{array}{l}1 \\
(1)^{*}\end{array}$ & $\begin{array}{l}\mathrm{g} 2 \\
(2)^{*}\end{array}$ & $\begin{array}{l}\text { g3 } \\
(3)^{*}\end{array}$ & $\begin{array}{l}\mathrm{g} \\
(4 \\
\end{array}$ & $\begin{array}{c}\text { g5 } \\
(5)^{*}\end{array}$ & $\begin{array}{l}\mathrm{h} 1 \\
(6)^{*}\end{array}$ & \begin{tabular}{|c|}
$\mathrm{h} 1$ \\
$(7)^{*}$
\end{tabular} & $\begin{array}{c}\text { h1 } \\
(8)^{*}\end{array}$ & $\begin{array}{l}\text { e1 } \\
(9)^{*} \\
\end{array}$ \\
\hline \multicolumn{12}{|l|}{ BIOFISICOS } \\
\hline Peña - Cerro Quininí & 16 & 60 & $\mathrm{R}$ & $\mathrm{B}$ & $\mathrm{Am}$ & $\mathrm{MA}$ & $\mathrm{SI}$ & $\mathrm{A}$ & $\mathrm{N}$ & $\mathrm{O}$ & $\mathrm{Mo}$ \\
\hline $\begin{array}{l}\text { Recorrido: Cambio uso del } \\
\text { suelo }\end{array}$ & 18 & 80 & $\mathrm{R}$ & B & $\mathrm{A}$ & $\mathrm{A}$ & SI & A & $\mathrm{N}$ & $\mathrm{O}$ & Mo \\
\hline $\begin{array}{l}\text { Paisaje: Vista Meseta de } \\
\text { Chinauta }\end{array}$ & 18 & 80 & B & B & $\mathrm{Am}$ & ---- & $\mathrm{SI}$ & $\mathrm{N}$ & $\mathrm{N}$ & $\mathrm{PO}$ & Mo \\
\hline Agroecosistemas & 18 & 60 & $\mathrm{~B}$ & $\mathrm{~B}$ & $\mathrm{Am}$ & Mo & --- & $\mathrm{A}$ & $\mathrm{N}$ & $\mathrm{O}$ & Mo \\
\hline $\begin{array}{l}\text { Senderismo: Recorrido Fauna } \\
\text { y Flora - Tangara Turquesa }\end{array}$ & 18 & 80 & B & B & Am & Mo & $\mathrm{N}$ & A & I & $\mathrm{O}$ & Mo \\
\hline Sendero: Cerro Quininí & 19 & 100 & $\mathrm{R}$ & $\mathrm{B}$ & $\mathrm{Am}$ & MA & $\mathrm{SI}$ & $\mathrm{A}$ & $\mathrm{I}$ & $\mathrm{O}$ & Mo \\
\hline Senderismo Antenas & 19 & 100 & $\mathrm{R}$ & $\mathrm{R}$ & $\mathrm{Am}$ & $\mathrm{A}$ & $\mathrm{SI}$ & $\mathrm{A}$ & A & $\mathrm{O}$ & Mo \\
\hline Bosque de Robles & 21 & 120 & $\mathrm{R}$ & B & $\mathrm{Am}$ & A & SI & A & I & $\mathrm{O}$ & Mo \\
\hline Cabeza del Indio & 21 & 120 & $\mathrm{R}$ & $\mathrm{B}$ & $\mathrm{Am}$ & A & $\mathrm{SI}$ & $\mathrm{A}$ & 1 & $\mathrm{O}$ & $\mathrm{Mo}$ \\
\hline \begin{tabular}{|l|} 
Pico del Águila \\
\end{tabular} & 22 & 120 & $\mathrm{~B}$ & $\mathrm{~B}$ & $\mathrm{Am}$ & A & $\mathrm{SI}$ & $\mathrm{A}$ & $\mathrm{I}$ & $\mathrm{O}$ & Mo \\
\hline \begin{tabular}{|l} 
Paisaje: Vista Panorámica \\
Valle del Magdalena \\
\end{tabular} & 19 & 100 & B & B & $\mathrm{Am}$ & Mo & $\mathrm{SI}$ & A & $\mathrm{N}$ & $\mathrm{O}$ & Mo \\
\hline Cueva del Mohán & 22 & 130 & $\mathrm{~B}$ & $\mathrm{~B}$ & $\mathrm{Am}$ & A & $\mathrm{SI}$ & $\mathrm{N}$ & $\mathrm{N}$ & $\mathrm{PO}$ & $\mathrm{S}$ \\
\hline
\end{tabular}




\begin{tabular}{|c|c|c|c|c|c|c|c|c|c|c|c|c|c|c|c|c|c|c|}
\hline \multicolumn{19}{|c|}{ CULTURALES Y ARQUEOLÓGICOS } \\
\hline \multicolumn{4}{|c|}{\begin{tabular}{|l|} 
Piedra del Palco: Petroglifo " \\
Las Estrellas"
\end{tabular}} & \multicolumn{2}{|l|}{16} & 60 & \multicolumn{2}{|c|}{$\mathrm{R}$} & B & \multicolumn{2}{|l|}{$\mathrm{Am}$} & MA & \multicolumn{2}{|l|}{$\mathrm{N}$} & A & I & $\mathrm{PO}$ & Mo \\
\hline \multicolumn{4}{|c|}{ Piedra del Palco } & \multicolumn{2}{|l|}{16} & 60 & \multicolumn{2}{|c|}{$\mathrm{R}$} & B & $\mathrm{Am}$ & & MA & \multicolumn{2}{|c|}{$\mathrm{N}$} & A & $\mathrm{I}$ & $\mathrm{PO}$ & Mo \\
\hline \multicolumn{4}{|c|}{$\begin{array}{l}\text { Piedra del Palco: Petroglifo " } \\
\text { El espiral" }\end{array}$} & \multicolumn{2}{|l|}{16} & 60 & \multicolumn{2}{|c|}{ AM } & B & $\mathrm{Am}$ & & MA & \multicolumn{2}{|l|}{$\mathrm{N}$} & A & 1 & $\mathrm{PO}$ & Mo \\
\hline \multicolumn{4}{|c|}{ Piedra del Gritadero } & \multicolumn{2}{|l|}{21} & 120 & \multicolumn{2}{|c|}{$\mathrm{R}$} & $\mathrm{B}$ & $\mathrm{Am}$ & & MA & \multicolumn{2}{|c|}{$\mathrm{SI}$} & A & 1 & $\mathrm{O}$ & Mo \\
\hline \multicolumn{4}{|c|}{$\begin{array}{l}\text { Piedra del Parto: "El } \\
\text { Nacimiento" }\end{array}$} & \multicolumn{2}{|l|}{19} & 100 & \multicolumn{2}{|c|}{$\mathrm{R}$} & B & $\mathrm{Am}$ & & MA & \multicolumn{2}{|c|}{ SI } & A & I & $\mathrm{O}$ & Mo \\
\hline \multirow{2}{*}{\multicolumn{4}{|c|}{$\begin{array}{l}\text { Casa de Turismo: " La } \\
\text { Cumbre" } \\
\text { Hacienda Cafetera } \\
\end{array}$}} & \multicolumn{2}{|l|}{19} & 100 & \multicolumn{2}{|c|}{$\mathrm{R}$} & $\mathrm{R}$ & A & & A & \multicolumn{2}{|c|}{ SI } & $\mathrm{A}$ & ---- & $\mathrm{SO}$ & Mo \\
\hline & & & & 16 & & 160 & \multicolumn{2}{|c|}{$M$} & $\mathrm{~B}$ & $\mathrm{Am}$ & & MA & \multicolumn{2}{|l|}{$\mathrm{S}$} & 1 & A & $\mathrm{PO}$ & $\mathrm{S}$ \\
\hline \multicolumn{2}{|l|}{$(1)^{*}$} & \multicolumn{2}{|l|}{$(2)^{*}$} & \multicolumn{2}{|l|}{$(3)^{*}$} & \multicolumn{2}{|l|}{$(4)^{*}$} & $(5$ & & $(6)^{*}$ & & & & & $(8)^{*}$ & & $(9)^{*}$ & \\
\hline Excelente & $E$ & Excelente & $E$ & Amplio & Am & Muy Alta & MA & $\mathrm{SI}$ & $\mathrm{S}$ & Suficiente & $\mathrm{S}$ & Sufic & & $\mathrm{S}$ & \begin{tabular}{|c|} 
Muy \\
Organizada \\
\end{tabular} & $\mathrm{MO}$ & $\begin{array}{l}\text { Muy } \\
\text { seguro }\end{array}$ & M \\
\hline Bueno & B & Bueno & B & Moderado & Mo & Alta & A & $\mathrm{NO}$ & $\mathrm{N}$ & Alguna & A & Alg & & A & Organizada & $\mathrm{O}$ & Seguro & $\mathrm{S}$ \\
\hline Regular & $R$ & Regular & $R$ & Poco & $\mathrm{P}$ & Moderado & Mo & & & Insuficiente & 1 & Insufi & & I & \begin{tabular}{|c|} 
Poco \\
Organizada \\
\end{tabular} & $\mathrm{PO}$ & Moderado & Mo \\
\hline Malo & $M$ & Malo & $M$ & Ninguno & $\mathrm{N}$ & Baja & $\mathrm{Ba}$ & & & Ninguna & $\mathrm{N}$ & Nin & & $\mathrm{N}$ & \begin{tabular}{|c|} 
Sin \\
organización
\end{tabular} & SO & & \\
\hline
\end{tabular}

Fuente: elaboración propia a partir de Enríquez et al. (2010) y Franco-Maass et al. (2009).

\section{Valores de la evaluación multicriterio total}

En la tabla 5 la evaluación multicriterio total de los recursos turísticos biofísicos presenta que el Pico del Águila es el recurso que mayor peso obtuvo, orden de preferencia 1 y valor final de ponderación de 6,53, por ende, mayor potencial y preferencia para ser ofrecido dada la importancia en las características de los criterios intrínsecos.

En la matriz de evaluación multicriterio total de los recursos turísticos culturales y arqueológicos (tabla 6) La Piedra del Gritadero es el recurso ecoturístico que mayor peso presentó, orden de preferencia 1 y valor final de ponderación 6,27.

Así las cosas, los recursos biofísicos, culturales y arqueológicos que tienen alta vocación turística por su mayor peso son el Pico del Águila (biofísico) y la Piedra del Gritadero (arqueológico y cultural).

\section{Perfil del visitante}

\section{Descripción de la demanda}

Del total de los encuestados el 64 \% correspondió a hombres y el 34,2 \% a mujeres, cuyo nivel de educación más representativo fue la formación universitaria con un $56,5 \%$ y $42,1 \%$, respectivamente, seguido del grupo de personas con estudios de bachillerato. Se observó que la formación posgradual en ambos géneros es baja, del $8,7 \%$ y el $10,5 \%$. La variable edad arrojó resultados que reflejaron que el 51,4\% de los encuestados es un grupo que osciló entre los 16 y 25 años, el 21,6 \% entre 26 y 35 años y el 11,7 \% entre 36 y 45 años. Es decir, el $84,7 \%$ se encuentran en un rango de edad entre los 16 y 45 años de edad lo que indica que el perfil de los visitantes es joven y joven-adulto.

Según el informe de OMT y WYSE (2012) el turismo de jóvenes es uno de los mercados del sector turístico que crece con más rapidez y presenta un mayor dinamismo. Se observó que los intervalos de edades más bajos, 5,4 y 3,6 \%, corresponde al grupo de visitantes mayores de 55 años, en este último puede considerarse que las condiciones de acceso son una de las causas por las cuales los turistas de estos intervalos de edad no son visitantes frecuentes; ellos se interesan más por caminar y escuchar charlas, se enfocan en participar en actividades de aprendizaje y prefieren estar en hoteles que acampar (Eagles, 2004). 
Tabla 5. Matriz de evaluación multicriterio total recursos turísticos biofísicos.

\begin{tabular}{|l|c|c|c|c|c|c|}
\hline \multicolumn{1}{|c|}{ Recursos Biofísicos } & $\begin{array}{c}\text { Suma lineal } \\
\text { de recursos } \\
\text { intrínsecos }\end{array}$ & $\begin{array}{c}\text { Suma lineal } \\
\text { de recursos } \\
\text { extrínsecos }\end{array}$ & $\begin{array}{c}\text { Suma } \\
\text { ponderada } \\
\text { de criterios } \\
\text { intrínsecos }\end{array}$ & $\begin{array}{c}\text { Suma ponderada } \\
\text { de criterios } \\
\text { extrínsecos }\end{array}$ & $\begin{array}{c}\text { Valor final } \\
\text { ponderaciones }\end{array}$ & $\begin{array}{c}\text { Orden de } \\
\text { preferencia }\end{array}$ \\
\hline Pico del Águila & 44,50 & 53,50 & 2,97 & 3,56 & 6,53 & 1 \\
\hline Peña_Cerro Quininí & 54,25 & 51,00 & 3,10 & 3,40 & 6,50 & 2 \\
\hline Cueva del Mohán & 51,75 & 51,00 & 2,96 & 3,40 & 6,35 & 3 \\
\hline Sendero: Cerro Quininí & 51,75 & 49,00 & 2,96 & 3,26 & 6,22 & 4 \\
\hline Bosque de Robles & 47,75 & 51,75 & 2,73 & 3,45 & 6,18 & 5 \\
\hline $\begin{array}{l}\text { Paisaje: Panorámica Valle del } \\
\text { Magdalena }\end{array}$ & 46,50 & 52,50 & 2,66 & 3,50 & 6,16 & 6 \\
\hline Cabeza del Indio & 47,00 & 52,00 & 2,69 & 3,46 & 6,15 & 7 \\
\hline Agrosistemas & 49,75 & 48,50 & 2,84 & 3,23 & 6,07 & 8 \\
\hline Recorrido Cambio Uso del Suelo & 45,75 & 51,00 & 2,61 & 3,40 & 6,01 & 9 \\
\hline Paisaje: Vista Meseta de Chinauta & 50,50 & 45,75 & 2,89 & 3,05 & 5,93 & 10 \\
\hline Recorrido_Tangara turquesa & 48,50 & 46,50 & 2,77 & 3,10 & 5,87 & 11 \\
\hline Senderismo: antenas & 46,75 & 44,25 & 2,67 & 2,95 & 5,62 & 12 \\
\hline
\end{tabular}

Fuente: elaboración propia a partir de Enríquez et al. (2010) y Franco-Maass et al. (2009).

Tabla 6. Matriz de evaluación multicriterio total recursos turísticos culturales y arqueológicos.

\begin{tabular}{|l|c|c|c|c|c|c|}
\hline \multicolumn{1}{|c|}{$\begin{array}{c}\text { Recursos culturales y } \\
\text { arqueológicos }\end{array}$} & $\begin{array}{c}\text { Suma lineal } \\
\text { de recursos } \\
\text { intrínsecos }\end{array}$ & $\begin{array}{c}\text { Suma lineal } \\
\text { de recursos } \\
\text { extrínsecos }\end{array}$ & $\begin{array}{c}\text { Suma } \\
\text { ponderada } \\
\text { de criterios } \\
\text { intrínsecos }\end{array}$ & $\begin{array}{c}\text { Suma ponderada } \\
\text { de criterios } \\
\text { extrínsecos }\end{array}$ & $\begin{array}{c}\text { Valor final } \\
\text { ponderaciones }\end{array}$ & $\begin{array}{c}\text { Orden de } \\
\text { preferencia }\end{array}$ \\
\hline Piedra del Gritadero & 48,75 & 52,25 & 2,78 & 3,48 & 6,27 & 1 \\
\hline Piedra del Parto & 47,75 & 50,50 & 2,73 & 3,37 & 6,09 & 2 \\
\hline $\begin{array}{l}\text { Casa de Turismo " La } \\
\text { Cumbre" }\end{array}$ & 46,75 & 49,50 & 2,67 & 3,30 & 5,97 & 3 \\
\hline Hacienda Cafetera & 47,25 & 47,38 & 2,70 & 3,16 & 5,86 & 4 \\
\hline $\begin{array}{l}\text { Petroglifo piedra del palco: " } \\
\text { El espiral" }\end{array}$ & 43,75 & 48,50 & 2,50 & 3,23 & 5,73 & 5 \\
\hline Piedra del Palco & 47,50 & 45,25 & 2,71 & 3,02 & 5,73 & 6 \\
\hline $\begin{array}{l}\text { Petroglifo piedra del palco: " } \\
\text { Las Estrellas" }\end{array}$ & 44,00 & 45,50 & 2,51 & 3,03 & 5,55 & 7 \\
\hline
\end{tabular}

Fuente: elaboración propia a partir de Enríquez et al. (2010) y Franco-Maass et al. (2009).

En la relación edad frente a la ocupación se encontró que el grupo más representativo es la edad de los 15 años, cuya ocupación corresponde a estudiantes. Seguida del grupo de personas entre 35 y 46 años con ocupaciones como contadores, ingenieros, economistas, entre otros. El ingreso mensual de los visitantes registró que $42,6 \%$ de los encuestados tienen ingresos bajos, entre uno y dos millones de pesos, el 37,2 \% tiene ingresos muy bajos que están por debajo del millón de pesos.
El 14,9\% percibe ingresos medios que están entre dos y tres millones de pesos. Los intervalos de ingresos altos y muy altos se encuentran en el $2,1 \%$ y $3,2 \%$ entre 3 y 4 millones y más.

En cuanto la relación lugar de alojamiento frente a la edad, se encontró que el campamento es la forma más habitual de hospedaje. Con respecto a la organización del viaje, el 65,5 \% de los encuestados respondieron que lo habían hecho de iniciativa propia, el 2,8\% a través del colegio o 
la universidad, las agencias de viajes ocupan una menor proporción. Respecto a los motivos de viaje la aventura fue el más representativo en un $43,9 \%$, seguido de caminar $14 \%$, acampar 7,5\%, investigar $3,7 \%$ y varias (donde se integran todas la anteriores) $20,6 \%$.

De la percepción de los servicios turísticos prestados el $17,1 \%$ de los visitantes informa que los servicios que recibieron fueron excursiones, el $14,3 \%$ comidas, el 11,4\% alojamiento y transporte respectivamente, un $40 \%$ manifestó recibir varios de los servicios antes mencionados. Manifiestan que el personal de los operadores que llegan a la reserva y que transmiten la información en términos de conocimiento idóneo: el 59 \% de los encuestados contestaron que sí lo hacían de manera adecuada y el $41 \%$ contestó que no lo hacían. Se hizo una comparación entre las variables nivel de educación, con respecto a la percepción sobre el servicio del personal que proviene de operadores sea idóneo; esto evidenció, en términos representativos, que los grupos de formación universitaria y posgradual consideran en un alto porcentaje que el personal proveniente de operadores no transmite información adecuada. El 50 \% de los encuestados manifestaron que el personal de la comunidad local presta el servicio de información de la reserva entre excelente y bueno y el $28 \%$ es regular, mientras que el restante lo considera malo y deficiente.

A la pregunta sobre si la infraestructura es adecuada para la atención a los turistas solo el 40,4 \% consideró que era buena y el 1,8 \% manifestó que es deficiente. En el caso de las vías de acceso, el $35,5 \%$ considera que son buenas, un $22,7 \%$ que son excelentes, mientras que más del $40 \%$ consideran que se encuentran entre regulares $y$ deficientes.

Finalizando con las preguntas de la encuesta, sobre qué actividades prioritarias para el manejo del área protegida y cuánto estaría dispuesto a pagar para ingresar al parque, a la primera pregunta el 19,6 \% de los encuestados respondieron que la disposición de residuos sólidos, 10,3\% adecuación de senderos, 2,8 \% personal, 1,9\% infraestructura, el 26,2 \% todas las anteriores y $39,3 \%$ otras no relacionadas. En la segunda pregunta resultó que aproximadamente el $30 \%$ entre hombres y mujeres encuestados dicen no asumir ningún costo para ingresar a la RFPCQ, mientras que el $25 \%$ de los hombres y mujeres encuestados consideran que sería $\$ 5000$ (cinco mil pesos colombianos).

\section{Estrategias: análisis Dofa}

Por medio del análisis Dofa se presentan estrategias como:

- Promover la elaboración del plan de manejo del área protegida en el que se establezcan las directrices para el desarrollo de programas de conservación y preservación de los recursos turísticos teniendo en cuenta procesos sostenibles.

- Capacitación para la interpretación ambiental.

- Integración directa de los turistas con el área protegida según el perfil de visitante.

- Mejorar la infraestructura y la planta física en la que se pueda prestar servicios a los turistas y orientar la visita de acuerdo con las preferencias de los diferentes grupos de perfiles que visitan la reserva: jóvenes, jóvenes adultos, personas mayores.

\section{Conclusiones}

Como parte del diagnóstico para la implementación del plan de manejo del turismo en las zonas de uso público de la RFPCQ, se logró la identificación y evaluación tanto de los recursos turísticos como del perfil del visitante. El trabajo con los expertos locales y la comunidad de la zona permitió identificar 19 recursos turísticos y clasificarlos en biofísicos, culturales y arqueológicos, a los cuales se les asignó criterios intrínsecos y extrínsecos, con el fin de evaluar la importancia que presentan y ofrecen en la actividad económica turística. 
Se evidenció que los recursos biofísicos que presentan mayor peso son: el Pico del Águila, Peña Cerro Quininí, la Cueva del Mohán y el Bosque de Robles. En tanto los recursos culturales y arqueológicos que presentaron más peso fueron la Piedra del Gritadero y la Piedra del Parto; aunque la Piedra del Palco en el análisis multicriterio no presentó una alta puntuación, sí es considerada por los lugareños como uno de los recursos más representativos por su importancia cultural y arqueológica.

Definir el perfil del visitante permitió obtener información de las personas que realizan turismo en la RFPCQ. Con base en lo anterior, se establecieron algunas estrategias que se obtienen del análisis Dofa, dentro de las cuales se destaca el desarrollo de programas de conservación y preservación de los recursos turísticos que se ofertan, capacitación cultural y arqueológica, los cultivos y la historia de la cultura indígena. Por otro lado, se hace necesario establecer puntos de información que cuenten con la infraestructura y planta física adecuada con el fin de orientar la visita de acuerdo con las preferencias de los diferentes grupos de perfiles. De esta forma, se aporta con la presente investigación en el diagnóstico en la fase de la planeación del turismo y así articular lo social, lo ambiental y lo económico en un proceso sostenible en que todas las partes obtengan ganancia sin afectar los ecosistemas.

\section{Agradecimientos}

A los docentes de la Facultad del Medio Ambiente y al Centro de Investigaciones y Desarrollo Científico de la Universidad Distrital Francisco José de Caldas por su apoyo económico. A los expertos locales de la comunidad que residen en la RFPCQ por su acompañamiento en los diferentes recorridos y disposición en las actividades que se elaboraron para el levantamiento del inventario y caracterización de los recursos turísticos.

\section{Referencias}

Alcaldía de Tibacuy (s. f). Diagnóstico territorial elementos constitutivos del espacio municipal. http://www.tibacuy-cundinamarca.gov.co/apc-aa-files/66346361613663353931303962633432/ DIAGNOSTICO TERRITORIAL.pdf

Angulo, G. (2002). El espacio turístico y las zonas y centros de interés turístico nacional. Universidad Austral de Chile, Escuela de Turismo. http://www.gestionturistica.cl/biblioteca/tesis/ pregrado/uach/doc/ga cuerpo.pdf

Boullón, R. (1978). El sistema turístico. OCEA/CICATUR. México: Ed. Trillas.

Cárdenas, D.; Salinas, N. R, (2007). Libro Rojo Pl. Colombia 4(1), 1-232. https://sinchi.org.Co/files/publicaciones/publicaciones/pdf/LR MADERABLES.pdf

Congreso de la República de Colombia (1996). Ley 300. Por la cual se expide la ley general de turismo y se dictan otras disposiciones. http:// www.alcaldiabogota.gov.co/sisjur/normas/ Norma1.jsp?i=8634

Corporación Autónoma Regional (CAR) (2012). Informe Técnico N. ${ }^{\circ} 131$. Bogotá: CAR.

Cubillos, O.: González, C.; Díaz E.; Ruiz, F.; Jiménez, Z. (2013). Guía para la planificación del ecoturismo en parques nacionales de Colombia. http://www.parquesnacionales.gov.co/ portal/wp- content/uploads/2014/05/guia planificacion ecoturismo pnn.pdf

De la Fuente, F. S. (2011). Análisis de correspondencias múltiple y simples. Madrid: Universidad Autónoma de Madrid. http://www. fuenterrebollo.com/Economicas/ECONOMETRIA/REDUCIR-DIMENSION/CORRESPONDENCIAS/correspondencias.pdf

Dredge, D. (1999). Destination place planning and design. Annals of Tourism Research, 26(4), 772-791. $\quad$ https://doi.org/10.1016/ S0160-7383(99)00007-9

Eagles, P. J. (2004). Trends affecting tourism in protected areas. Ponencia presentada en Policies, 
methods and tools for visitor managementproceedings of the second international conference on monitoring and management of visitor flows in recreational and protected areas (pp. 18-26), Rovaniemi, Finland.

Enríquez, M.; Osorio, M.; Franco-Maass, S.; Ramírez, I.; Nava, G. (2010). Evaluación multicriterio de los recursos turísticos del Parque Estatal Sierra de Nanchititla; Estado de México. El Periplo Sustentable, 18. https://www.redalyc.org/articulo.oa?id=193414423001

Franco-Maass, S.; Osorio-García, M.; Nava-Bernal, G.; Regil-García, H. H. (2009). Evaluación multicriteriodelos recursosturísticos: ParqueNacional Nevado de Toluca. Estudios y Perspectivas en Turismo, 18, 208-226. http://ri.uaemex.mx/ bitstream/handle/20.500.11799/40084/Evaluaci $\%$ C3\%B3n\%20multicriterio $\% 20 \mathrm{de} \% 20$ los $\% 20$ recursos $\% 20$ tur $\%$ C3\%ADsticos $\% 20$ $\% 20$ Parque $\% 20$ Nacional $\% 20$ Nevado $\% 20$ de\%20Toluca\%20-\%20M\%C3\%A9xico. pdf? sequence $=1$

Hernández, X. (1977). Metodología para el estudio de agroecosistemas con persistencia de tecnología agrícola tradicional. En Agroecosistemas en México (pp. 321-333). México D. F.: Colegio de posgraduados-ENA.

Holdridge, L. R. (1947). Determination of World Plant Formations From Simple Climatic Data. Science. 105(2727), 367-368. https://doi. org/10.1126/science.105.2727.367

Jarvis, D.; Myer, L.; Klemick, H.; Guarino, L.; Smale, M.; Brown, A. Hodgkin (2006). Guía de capacitación para la conservación in Situ en Fincas. Instituto Internacional de Recursos Fitogenéticos (IPGRI). Material producido con apoyo del Instituto Nacional de Investigación y Tecnología Agraria y Alimentaria de España. https://www.bioversityinternational.org/fileadmin/ migrated/uploads/ tx news/Material de apoyo a la capacitaci\%C3\%B3n en conservaci\%C3\%B3n In $\underline{\text { Situ de la diversidad vegetal en areas pro- }}$ tegidas y en fincas 905.pdf
Jiménez B, L. H.; Jiménez, W. G. (2013). Turismo: tendencias globales y planificación estratégica. Bogotá: Ecoe Ediciones. https://www.ecoeediciones.com/wp-content/uploads/2015/08/Turismo.-tendencias-globales.pdf

Ministerio de Comercio Industria y Turismo (2013). Asistencia técnica en planificación del turismo. Bogotá: Ministerio de Comercio Industria y Turismo.

Ministerio del Medio Ambiente y Desarrollo Sostenible (2012). Portal web. http://www.minambiente.gov.co/.

OMT; Wyse, T. C. (2012). La fuerza del turismo joven. Informe del programa de miembros afiliados. España: OMT. http://cf.cdn.unwto.org/ sites/all/files/pdf/lafuerzadelturismojoven_ omt2011_esp_0.pdf

Ordoñez J. C.; Montes, C. R. (2013). Orquideoflora de la reserva natural Quininí, Tibacuy, Cundinamarca, Colombia y consideraciones para su bioprospección. Revista Colombiana de Orquideología, 1, 18-33. http://revista.sco.org. co/index.php/orquideologia/article/view/2

Organización Mundial de Turismo (OMT) (1998). Introducción al turismo. España: Organización Mundial de Turismo. http://dspace. universia.net/bitstream/2024/1043/1/INTRODUCCION+AL+TURISMO +OMT.pdf

Pinzón, V. (2010). Diseño, análisis de capacidad de carga e implementación ambiental para el sendero ecoturistico Cerro Quininí en el municipio de Tibacuy, Cundinamarca. Tesis, Universidad Distrital Francisco José de Caldas, Bogotá.

Salas, R.; Tapias, M. (2000). Tibacuy, Un sitio arqueológico de frontera entre grupos indígenas del altiplano Cundiboyacense y el valle Medio del Magdalena. Boletín de Arqueología, Fian, 15(2). https://publicaciones.banrepcultural. org/index.php/fian/article/view/6077/6294

SECTUR (2004). Planeación y Gestión del Desarrollo Turístico Municipal. México D. F.: SECTUR. https://cedocvirtual.sectur.gob.mx/ janium/Documentos/003162Pri0000.pdf 
SECTUR (2005). Informe Turismo en México.

Seixas, C. (2005). A bordagens eTécnicas de Pesquisa Participativaem Gestão de Recursos Naturais. En P. Freire, B. Fikret y C. Seixas (eds.), Integrada e Participativa de Recursos Naturais: conceitos, métodos e experiências. Florianópolis: Secco/ APED. https://www.worldcat.org/title/gestao-integrada-e-participativa-de-recursos-naturais-conceitos-metodos-e-experiencias/ oclc/71310852

Torrejón, A. (2006). Una herramienta clásica, pero aún hoy de uso poco frecuente. Los análisis F.O.D.A. en el turismo. Semanario Region ${ }^{\circledR}$. Del 4 al 10 de agosto, año 16, n. ${ }^{\circ}$ 768. R.N.P.I. n. ${ }^{\circ}$ 359581. Santa Rosa, Provincia de La Pampa, Argentina. http://www.region.com.ar/productos/semanario/archivo/768/turismo768.htm
Vargas, G. E. (1992). Análisis y clasificación del uso y cobertura de la tierra con interpretación de imágenes. Bogotá: Instituto geográfico Agustín Codazzi. http://documentacion.ideam.gov. co/cgi-bin/koha/opac-detail.pl?biblionumber $=907 \&$ shelfbrowse itemnumber $=949$

Vásquez, V. (2005). Reservas forestales protectoras de Colombia: Atlas Básico. Bogotá: Instituto Geográfico Agustín Codazi. http://documentacion.ideam.gov.co/cgi-bin/koha/opac-detail. pl?biblionumber $=15240$

Zamorano, F. (2002). Turismo alternativo. Servicios turísticos diferenciados. Trillas. México. https://www.entornoturistico.com/wp-content/ uploads/2017/03/Turismo-Alternativo-Servicios-Tur\%C3\%ADsticos-Diferenciados-de-Francisco-M.-Zamorano.pdf 